\title{
NOTE ON THE IDEALS OF CYCLIC ALGEBRAS*
}

\section{BY RALPH HULL}

1. Introduction. The purpose of this note is the generalization of certain results in a recent paper by Latimer $\dagger$ on the number of ideals of given norm in a generalized quaternion algebra.

We consider rational cyclic division algebras $D$ of prime degree $n(\geqq 2)$ over the field $R$ of rational numbers. Let $\mathfrak{D}$ be any maximal order $\ddagger$ of $D$. The reduced discriminant of $\mathfrak{D}$ is an invariant $\Delta=\Delta(D)$ of $D$ called the discriminant of $D$, and is of the form $\Delta= \pm \sigma^{n(n-1)}$, where $\sigma=q_{1} \cdots q_{s}$ is the product of the distinct rational primes $q_{1} \cdots q_{s}$ which are ramified $\S$ in $D$. For each such $q$, the two-sided ideal $q \mathfrak{v}$ is the $n$th power of an indecomposable two-sided prime ideal of $\mathfrak{b}$, and the $q$-adic extension $D_{q}$ is a division algebra of degree $n$ of $R_{q}$. For all other rational primes $p, D_{p}$ is the algebra of all matrices of degree $n$ over $R_{p}$ and $\mathrm{o} p$ is a two-sided prime ideal of $\mathrm{o}$ having only one-sided ideal divisors.

By a (normal) ideal of $D$ is meant an ideal (one or two-sided) with respect to some maximal order of $D$. Such an ideal is called integral if it is contained in its right or left order. We denote various maximal orders by $\mathfrak{D}, \mathfrak{D}_{1}, \mathfrak{D}_{2}, \cdots$, and an ideal $\mathfrak{a}$ by $\mathfrak{a}_{i j}$ if $\mathfrak{D}_{i} \mathfrak{a}=\mathfrak{a} \mathfrak{o}_{j}=\mathfrak{a}$ and it is necessary to indicate $\mathfrak{D}_{i}$ and $\mathfrak{D}_{j}$. The (reduced) norm of an ideal is an ideal of $R$ such that, for a principal ideal $\alpha \mathfrak{v}($ or $\mathfrak{D} \alpha), \alpha$ in $D, N(\alpha \mathfrak{v})($ or $N(\mathfrak{D} \alpha))=(N(\alpha))$, where $N(\alpha)$ is the reduced norm corresponding to the rank equation of degree $n$. Our object is to prove the following result.

Theorem. Let o be any maximal order of $D$ and let $A$ be any rational integer. Write $A=A_{1} A_{0}$, where $A_{0}$ is prime to $\Delta(D)$ and every prime factor of $A_{1}$ divides $\Delta(D)$. Then the number of integral

* Presented to the Society, April 9, 1937.

† C. G. Latimer, Transactions of this Society, vol. 40 (1936), pp. 439-450.

$¥$ Maximal orders for all $D$ have been given explicitly. See Albert, this Bulletin, vol. 40 (1934), pp. 164-176, for $n=2$, and Hull, Transactions of this Society, vol. 38 (1935), pp. 515-530, for $n>2$.

$\S$ We refer to Deuring, Algebren, Ergebnisse der Mathematik, Chapter VI, for all definitions and theorems used here except when explicit reference elsewhere is given. 
D-right ideals of norm $(A)$ is equal to the number of classes of right associated integral matrices of degree $n$ and determinant $A_{0}$.

Two integral matrices $M_{1}$ and $M_{2}$ are said to be right asso-

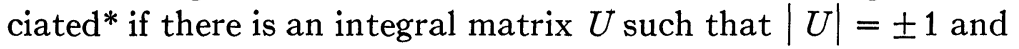
$M_{2}=M_{1} U$. In case $n=2$, the number of such classes of matrices of given determinant $A_{0}$ is easily seen to be the sum of the divisors $\dagger$ of $A_{0}$.

2. Preliminary Lemmas. We need two lemmas easily obtained from the general ideal theory of linear algebras (Deuring, loc. cit.).

Let $\mathfrak{a}=\mathfrak{a}_{r+1,1}$ be an integral ideal and let

$$
N(a)=(A), \quad A=p_{r}^{\gamma_{r}} \cdots p_{1}^{\gamma_{1}},
$$

where the $p$ 's are distinct rational primes.

Lemma 1. The ideal $\mathfrak{a}=\mathfrak{a}_{r+1,1}$ has a special factorization

$$
\mathfrak{a}=\mathfrak{a}_{r+1, r}^{(r)} \cdot \mathfrak{a}_{r, r-1}^{(r-1)} \cdots \mathfrak{a}_{2,1}^{(1)}, \mathfrak{a}^{(i)} \text { integral, } N\left(\mathfrak{a}^{(i)}\right)=p_{i}^{\gamma_{i}} .
$$

For a given order $p_{r}, \cdots, p_{1}$ of the distinct prime divisors of $A$ in (1), the special factorization (2) is unique.

The existence of (2) is implied by the fact that there exists a factorization of $\mathfrak{a}$ into indecomposable ideals in which the order of the prime ideals to which the factors belong is arbitrarily assigned (Deuring, p. 106). To prove the uniqueness claimed we consider $p$-components and apply a theory due to Hasse (Deuring, pp. 94-107).

Let $p$ be any rational prime. Then from (2)

$$
(\mathfrak{a})_{p}=\left(\mathfrak{a}^{(r)}\right)_{p} \cdots\left(\mathfrak{a}^{(1)}\right)_{p},
$$

where $(\mathfrak{a})_{p}$ denotes the $p$-component, that is, $p$-adic limit set of a. If $(p, A)=1,(\mathfrak{a})_{p}$ is a maximal order of $D_{p}$, and since $\mathfrak{b}_{r+1} \mathfrak{a}=\mathfrak{a b}_{1}=\mathfrak{a}$, we have $(\mathfrak{a})_{p}=\left(\mathfrak{b}_{r+1}\right)_{p}=\left(\mathfrak{o}_{1}\right)_{p}$. Similarly $\left(\mathfrak{a}^{(1)}\right)_{p}$ $=\left(\mathfrak{D}_{2}\right)_{p}=\left(\mathfrak{D}_{1}\right)_{p},\left(\mathfrak{a}^{(2)}\right)_{p}=\left(\mathfrak{b}_{3}\right)_{p}=\left(\mathfrak{D}_{2}\right)_{p}=\left(\mathfrak{b}_{1}\right)_{p}$, and so on, and it is clear that $\left(\mathfrak{a}^{(i)}\right)_{p}=(\mathfrak{a})_{p},(i=1, \cdots, r)$. If $p=p_{i},(i=1, \cdots, r)$, III.

* MacDuffee, The Theory of Matrices, Ergebnisse der Mathematik, Chapter

† See Latimer, loc. cit. 
we find in a similar way that $\left(\mathfrak{a}^{(r)}\right)_{p}=\cdots=\left(\mathfrak{a}^{(i+1)}\right)_{p}=\left(\mathfrak{b}_{r+1}\right)_{p}$ and $\left(\mathfrak{a}^{(i-1)}\right)_{p}=\cdots=\left(\mathfrak{a}^{(1)}\right)_{p}=\left(\mathfrak{o}_{1}\right)_{p}$, whence $\left(\mathfrak{a}^{(\imath)}\right)_{p}=(\mathfrak{a})_{p}$. Hence, for every rational prime $p$, the $p$-component of each $\mathfrak{a}^{(i)}$ is uniquely determined by $\mathfrak{a}$. It follows that each $\mathfrak{a}^{(i)}$ is uniquely determined by $\mathfrak{a}$ since each is determined by the totality of its $p$-components.

Lemma 2. If $p$ is ramified in $D$, there is exactly one ideal of $D_{p}$ of given norm $p^{\nu}$. If $p$ is not ramified in $D$, the number of right ideals of $D_{p}$ with respect to a given maximal order of $D_{p}$, of given norm $p^{\nu}$, is the number $\psi\left(p^{\nu}\right)$ of classes of right associated rational integral matrices of degree $n$ and determinant $p^{\nu}$.

For the first part of Lemma 2 we have only to note that every ideal of $D_{p}, p$ ramified in $D$, is a power of the single prime ideal of the unique maximal order of $D_{p}$. The second part is seen as follows. A maximal order $\mathfrak{o}_{p}$ of $D_{p}, p$ not ramified in $D$, is of the form $\mathfrak{o}_{p}=\sum c_{i j} \mathfrak{g}_{p}$, where the $c_{i j},(i, j=1, \cdots, n)$, are matrix units and $\mathfrak{g}_{p}$ is the maximal order of $R_{p}$. Every $\mathfrak{o}_{p}$-right ideal is a principal ideal $\alpha 0_{p}$, where $\alpha$ is of the form (Deuring, loc. cit. p. 101)

(3) $\alpha=p^{\mu_{1}} c_{11}+d_{21} c_{21}+p^{\mu_{2}} c_{22}+\cdots+d_{n 1} c_{n 1}+\cdots+p^{\mu_{n}} c_{n n}$,

where $\mu_{1}+\mu_{2}+\cdots+\mu_{n}=\nu$, and $d_{i j}$ is uniquely determined modulo $p^{\mu_{j}}$. The last part of the lemma is obvious from (3).

3. Proof of the Theorem. Since every integral ideal $a$ of norm $(A), A$ as in (1), has the unique special factorization (2), we proceed to count the number of possible distinct sets $\mathfrak{a}^{(1)}, \cdots, \mathfrak{a}^{(r)}$ which yield an $\mathfrak{a}$.

Consider first $\mathfrak{a}^{(1)}$ whose right order is the fixed maximal order $\mathfrak{p}_{1}=\mathfrak{b}$, of the theorem. For every rational prime $p \neq p_{1}$, we have $\left(\mathfrak{a}^{(1)}\right)_{p}=(\mathfrak{o})_{p}$, and for $p=p_{1},\left(\mathfrak{a}^{(1)}\right)_{p}$ is a right ideal with respect to $(\mathfrak{D})_{p}$ of norm $p_{1}{ }^{\nu_{1}}$. Thus $\left(\mathfrak{a}^{(1)}\right)_{p}$ is unique for all $p \neq p_{1}$ and, by Lemma 2, there are precisely 1 or $\psi\left(p_{1}^{\nu_{1}}\right)$ possibilities for $\left(\mathfrak{a}^{(1)}\right)_{p_{1}}$ according as $p_{1}$ is ramified or unramified in $D$. Hence by an argument used in the proof of Lemma 1, there are precisely 1 or $\psi\left(p_{1}^{\nu_{1}}\right)$ possibilities for the factor $\mathfrak{a}^{(1)}$ in the respective cases.

Suppose, next, that $\mathfrak{a}^{(1)}$ is fixed and consider $\mathfrak{a}^{(2)}$, whose right order $\mathbb{D}_{2}$ is uniquely determined by $\mathfrak{a}^{(1)}$, since $\mathfrak{D}_{2}$ is the left order of $\mathfrak{a}^{(1)}$. The same argument made for $\mathfrak{a}^{(1)}$ and $\mathfrak{o}_{1}$ applies to $\mathfrak{a}^{(2)}$ and $\mathfrak{D}_{2}$, and we can proceed similarly with $\mathfrak{a}^{(3)}, \mathfrak{a}^{(4)}, \cdots$, suc- 
cessively. It is plain that the total number of sets $\mathfrak{a}^{(1)}, \cdots, \mathfrak{a}^{(r)}$ is $\prod \psi\left(p_{j}{ }^{\nu_{j}}\right)$, where $j$ ranges over those of the integers $1, \cdots, r$ for which $p_{j}$ is unramified in $D$, that is $\Pi\left(p_{j}{ }^{\nu_{j}}\right)=A_{0}$. To complete the proof of the theorem we have to show that $\prod \psi\left(p_{j}{ }^{{ }_{j}}\right)=\psi\left(A_{0}\right)$. This follows from the following lemma.

Lemma 3. If $A=B C$, where $A, B, C$ are rational integers such that $(B, C)=1$, then $\psi(A)=\psi(B) \psi(C)$.

To prove this lemma we apply the methods of $\$ 2$ to the simple algebra $S$ of all rational matrices of degree $n$. For a given system of matrix units $c_{i j},(i, j=1, \cdots, n)$, the set $\mathfrak{m}=\sum c_{i j} \mathfrak{g}$, where $\mathfrak{g}$ denotes the set of rational integers, is a maximal order of $S$. Every integral $\mathrm{m}$-right ideal is a principal ideal (MacDuffee, loc. cit.) $\mathfrak{a}=M_{1} \mathfrak{m}$, where $M_{1}$ is an integral matrix and the reduced norm of $\mathfrak{a}$ is $\left(\left|M_{1}\right|\right)=(A)$, say. If also $\mathfrak{a}=M_{2} \mathfrak{m}$, then $M_{2}=M_{1} U, U$ integral, $|U|= \pm 1$. Hence $\psi(A)$ is the number of integral $\mathfrak{m}$-right ideals of norm $(A)$. In $S$, we have unique special factorizations* similar to those of Lemma 1 . Hence if $A=B C$, we can count the number of $\mathfrak{m}$-right ideals of norm $(A)$ by counting the number of right ideals, with respect to certain maximal orders of $S$, of norms $(B)$ and $(C)$. This yields the lemma.

4. Applications of the Theorem. In case the class number $h$ of $D$ is one, $\dagger$ our theorem yields interesting results concerning representations $\ddagger$ by the norm form associated with a maximal order $\mathfrak{D}$ of $D$. Thus, if $h=1$, every ideal of $D$ is principal, $\mathfrak{a}_{i j}=\alpha \mathfrak{o}_{j}=\mathfrak{v}_{i} \beta, \alpha, \beta$ in $D$. If also, $a_{i j}=\alpha^{\prime} \mathfrak{b}_{j}$, then $\alpha^{\prime}=\alpha u$, where $u$ is a unit of $\mathrm{D}_{j}$, and the norm form associated with $\mathrm{D}_{j}$ is universal. The number of sets of integral representations $\S$ of $A$ is $\psi\left(A_{0}\right)$. The representations of a set are connected by the automorphs of the form associated with the units of $\mathfrak{D}$.

UNIVERSITY OF MichigaN

* Every rational prime is unramified in $S$.

$\dagger$ M. Eichler, Journal für Mathematik, vol. 176 (1937), pp. 192-202, has proved general results on the class number of algebras which imply $h=1$ for all $D$ with $n>2$ and for rational quaternion algebras with real splitting fields.

$\ddagger$ Cf. L. E. Dickson, Algebren und ihre Zahlentheorie, $\$ 100$.

$\S$ We must have $A>0$ for $n=2, D$ definite. In this case, the infinite prime spot of $R$ is said to be ramified in $D$. 